\title{
Investigating the association between diabetes mellitus, depression and psychological distress in a cohort of South African teachers
}

\author{
A K Domingo, ${ }^{1} \mathrm{MB}$ ChB, FC Psych (SA); L Asmal, ${ }^{1} \mathrm{MB}$ ChB, FCPsych (SA), MMed (Psych), MSc Clin Epi; \\ S Seedat, ${ }^{1}$ MB ChB, FCPsych (SA), MMed (Psych), PhD; T M Esterhuizen, ${ }^{2}$ MSc (Epidemiology); C Laurence, ${ }^{2}$ MB ChB, MSc Clin Epi; \\ J Volmink, ${ }^{2,3}$ BSc, MB ChB, MPH, DPhil \\ ${ }^{1}$ Department of Psychiatry, Faculty of Medicine and Health Sciences, Stellenbosch University, Tygerberg, Cape Town, South Africa \\ ${ }^{2}$ Centre for Evidence-based Health Care, Faculty of Medicine and Health Sciences, Stellenbosch University, Tygerberg, Cape Town, South Africa \\ ${ }^{3}$ South African Cochrane Centre, South African Medical Research Council, Tygerberg, Cape Town, South Africa
}

Corresponding author: A K Domingo (abduldomingo@gmail.com)

\begin{abstract}
Background. Diabetes mellitus (DM) may increase the risk of depression as a result of a sense of threat of debilitating complications or because of associated lifestyle changes. Depression may increase the risk of type 2 diabetes as a result of poor health behaviours.

Objective. To determine the association between diabetes mellitus, depression and psychological distress in a cohort of South African (SA) teachers.

Methods. Teachers from 111 public schools in the Metro South District of the Cape Metropolitan area, SA, were invited to participate in this study. The Center for Epidemiologic Studies Depression Scale (CES-D) and the Kessler Psychological Distress Scale (K10) were used to assess depression and psychological distress, respectively. A professional nurse completed a physical examination and collected blood for measurement of glucose, cholesterol and serum creatinine.

Results. Of the 388 teachers who completed the questionnaires, $67.5 \%$ were female and the average age was 46.2 years (standard deviation 8.7). Psychological distress was identified in $28.1 \%$ of the cohort and depression in $15.5 \%$, and $7.7 \%$ were found to fulfil criteria for DM. A diagnosis of DM was associated with an increased risk of depression (adjusted odds ratio (AOR) 3.90; 95\% confidence interval (CI) 1.33 11.37) and psychological distress (AOR 3.62; 95\% CI 1.31 - 10.00).

Conclusion. The high prevalence of obesity and DM in this cohort of SA teachers is of concern. A diagnosis of DM was strongly associated with an increased risk of depression and psychological distress.
\end{abstract}

S Afr Med J 2015;105(12):1057-1060. DOI:10.7196/SAMJ.2015.v105i12.9843

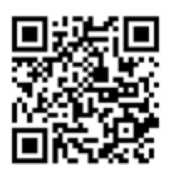

Diabetes mellitus (DM) and depression are highly prevalent conditions, and each contributes significantly to morbidity and mortality worldwide. The number of people with DM globally is projected to rise from 171 million in 2000 to 366 million in $2030,{ }^{[1]}$ and developing countries are likely to carry an increased share of this burden. While data are limited for South Africa (SA), there is evidence that this burden is already considerable, with the prevalence of type $2 \mathrm{DM}$ estimated at $28.2 \%$ in a recent community-based study in Bellville South, Cape Town. ${ }^{[2]}$ The estimated prevalence of major depressive episodes (MDE) in SA is also high, based on the findings of the South African Stress and Health (SASH) study. ${ }^{[3]}$ The prevalence of MDE in SA was found to be $9.7 \%$ and $4.9 \%$ for lifetime and a 12 -month period, respectively. ${ }^{[3]}$

Poor health behaviours associated with depression, such as smoking, physical inactivity and high caloric intake, may increase the risk of type $2 \mathrm{DM} \cdot{ }^{[4]}$ Depression is also associated with physiological abnormalities, including activation of the hypothalamic-pituitaryadrenal axis, sympathoadrenal system and proinflammatory cytokines, which can induce insulin resistance and contribute to diabetes risk. ${ }^{[4]}$ Furthermore, the sense of threat of debilitating complications and the lifestyle changes associated with management of diabetes may increase the risk of depression. ${ }^{[4]} \mathrm{A}$ meta-analysis of 39 studies reported that $11 \%$ of patients with DM met the criteria for comorbid major depressive disorder. ${ }^{[5]}$ Another meta-analysis that examined the relation between depression and diabetes onset found that the risk of developing type $2 \mathrm{DM}$ was $37 \%$ greater in depressed adults than in adults without depression. ${ }^{[6]}$
SA has a quadruple burden of disease: a combination of povertyrelated infectious diseases, violence-related injuries, perinatal and maternal disease, and an increase in lifestyle-related non-communicable diseases (NCDs). ${ }^{[7]}$ The potential link between depression, psychological distress and DM may therefore be particularly relevant and warrants further investigation.

SA teachers, in common with teachers everywhere, have high job stressors related to time pressures, the educational system, professional distress and learner misbehaviour. ${ }^{[8]}$ Job stress and lack of job satisfaction are associated with many stress-related illnesses, including hypertension, heart disease, stomach ulcers, asthma, mental distress and alcohol misuse. ${ }^{[8]}$

We investigated the association between DM, depression and psychological distress in a pilot cohort of SA teachers. Specific objectives of this study were to: (i) describe the demographic and clinical profile of the cohort; (ii) determine the prevalence of DM, depression, and psychological distress; and (iii) determine whether DM is associated with depression and psychological distress.

\section{Methods \\ Study design}

The study involved secondary analysis of SA baseline data from the Partnership for Cohort research and Training (PaCT) pilot study. The PaCT initiative was established to study NCDs in four African countries (SA, Nigeria, Tanzania and Uganda), and pilot studies were conducted to assess the feasibility of conducting longitudinal cohort studies at each site. The SA pilot study was conducted among teachers, 
and additional aims were to determine the prevalence of cardiovascular disease risk factors and chronic kidney disease.

\section{Participants}

The principals of 111 primary, secondary, and intermediate public schools where 20 or more teachers were employed in the Metro South District of the Cape metropolitan area were approached for permission for their schools to participate. All teachers from schools where principals indicated a willingness to participate were invited to participate. Teachers excluded from the study were those under the age of 18 years and those intending to retire or to relocate in the following 6-month period.

\section{Procedure}

School principals were tasked with distributing self-administered questionnaire packets and consent forms to teachers at their schools. Teachers returned the completed questionnaires and consent forms in a sealed envelope and were then seen by a nurse, who gathered select physical measurements and biological specimens.

\section{Measures}

The core questionnaire was developed in collaboration with Harvard University and universities from four African countries using the World Health Organization (WHO) stepwise approach to chronic disease risk factor surveillance (STEPS) as a template..$^{[9]}$ The questionnaire focused on demographic and health-related information such as age, gender, ethnicity, educational level, physical activity, chronic medication use, tobacco use, alcohol consumption, and personal and family medical history related to cardiovascular disease and other chronic NCDs.

The questionnaires in SA included the Center for Epidemiologic Studies - Depression Scale (CES-D) and the Kessler Psychological Distress Scale (K10) to determine the risk of depression and psychological distress, respectively. The CES-D is a validated selfreport screening instrument designed to measure the frequency and severity of depressive symptoms in community samples. It consists of 20 items, each rated on a 4-point scale. A CES-D score of $\geq 16$ was used as a cutoff point for the study and is correlated with mild to moderate depressive symptoms. ${ }^{[10]}$ The K10 is a self-report screening instrument that assesses anxiety and depressive symptoms over the preceding 4 weeks on a 10 -item scale. K10 total scores range from 10 to 50 , with clinically relevant psychological distress defined as a K10 score of $\geq 20$. ${ }^{[1]}$
DM was defined as a fasting ( $>8$ hours after a main meal) blood glucose concentration of $\geq 7.0 \mathrm{mmol} / \mathrm{L}$, or a random (approximately 2 hours after a main meal) blood glucose level $\geq 11.1 \mathrm{mmol} / \mathrm{L}$, or self-reported diabetes on insulin or oral hypoglycaemic drugs. A professional nurse completed a physical examination and recorded blood pressure (BP), weight, height and waist circumference. Glucose, total cholesterol, high-density lipoprotein cholesterol and serum creatinine were measured.

\section{Data analysis}

SPSS version 20 (IBM, USA) was used for analysis of data. A $p$-value of $<0.05$ was considered statistically significant. Bivariate associations between categorical exposures and outcomes were assessed using Pearson's $\chi^{2}$ tests or Fisher's exact tests, as appropriate. In the case of continuous predictor variables, $t$-tests were used if they were normally distributed and Mann-Whitney $U$-tests were used for non-parametric data. We used unadjusted and adjusted logistic regression to assess the association between DM and depression, and DM and psychological distress. We adjusted for the following potential confounders: age, gender, body mass index (BMI, $\mathrm{kg} / \mathrm{m}^{2}$ ), hypertension, serum cholesterol, and smoking. A backward stepwise model was constructed with entry and exit probabilities set at 0.1 and 0.05 , respectively. Odds ratios (ORs) and 95\% confidence intervals (CIs) are presented in the final model.

\section{Ethical considerations}

The institutional ethics committees of Stellenbosch and Harvard universities app- roved the study. Permission to conduct the study was obtained from the Western Cape Education Department and from the principal of each school. A letter was sent to the provincial offices of teacher trade unions to inform them of the study. All participants signed written informed consent and confidentiality was strictly maintained throughout the study. Teachers were not remunerated for participating in the study.

\section{Results \\ Demographic and clinical characteristics}

Fifty-nine of the 111 school principals (53.2\%) who were approached agreed to their schools participating in the study. A total of 489 teachers enrolled in the study, of whom 478 completed the baseline questionnaire. A further 90 participants were excluded because of missing mental health data. The cohort for this analysis comprised 388 participants with a mean (standard deviation (SD)) age of 46.2 (8.7) years (Table 1). There were more females $(n=262,67.5 \%)$ than males $(n=126,32.5 \%)$ in the sub-sample. The mean (SD) serum cholesterol was found to be 5.5 (1.1) $\mathrm{mmol} / \mathrm{L}$, BP 138.6/81.0 (19.5/11.3) $\mathrm{mmHg}$ and serum glucose $5.6(2.1) \mathrm{mmol} / \mathrm{L}$. Mean serum cholesterol and glucose did not differ significantly between male and female participants. The mean (SD) BMI in both female (31.2 (7.8)) and male (30.0 (5.9)) participants corresponded to the WHO obesity category. Male participants had a mean (SD) systolic BP of 145.5 (19.3) $\mathrm{mmHg}$ and diastolic BP of 83.9 (11.2) $\mathrm{mmHg}$, while female participants had significantly lower

\section{Table 1. Demographic and clinical characteristics}

\begin{tabular}{|c|c|c|c|c|}
\hline Variable & Total $(N=388)$ & Females $(n=262)$ & Males $(n=126)$ & $p$-value \\
\hline Age (years), mean (SD) & $46.2(8.7)$ & $46.1(8.5)$ & $46.4(9.2)$ & 0.707 \\
\hline Weight $(\mathrm{kg})$, mean $(\mathrm{SD})$ & $80.9(18.2)$ & $77.1(17.3)$ & $88.1(17.6)$ & $<0.001$ \\
\hline BMI $\left(\mathrm{kg} / \mathrm{m}^{2}\right)$, mean $(\mathrm{SD})$ & $30.7(7.2)$ & $31.2(7.8)$ & $30.0(5.9)$ & 0.151 \\
\hline $\begin{array}{l}\text { Serum cholesterol } \\
(\mathrm{mmol} / \mathrm{L}), \text { mean }(\mathrm{SD})\end{array}$ & $5.5(1.1)$ & $5.4(1.1)$ & $5.5(1.0)$ & 0.260 \\
\hline $\begin{array}{l}\text { Serum glucose }(\mathrm{mmol} / \mathrm{L}) \text {, } \\
\text { mean }(\mathrm{SD})\end{array}$ & $5.6(2.1)$ & $5.5(1.7)$ & $5.9(2.8)$ & 0.186 \\
\hline $\begin{array}{l}\text { Systolic BP (mmHg), mean } \\
\text { (SD) }\end{array}$ & $138.6(19.5)$ & $135.3(18.7)$ & $145.5(19.3)$ & $<0.001$ \\
\hline $\begin{array}{l}\text { Diastolic BP }(\mathrm{mmHg}) \text {, } \\
\text { mean (SD) }\end{array}$ & $81.0(11.3)$ & $79.5(11.1)$ & $83.9(11.2)$ & $<0.001$ \\
\hline Current smoking, $n(\%)$ & $74(19.8)$ & $40(16.1)$ & $34(27.4)$ & 0.010 \\
\hline $\begin{array}{l}\text { K10, median (interquartile } \\
\text { range) }\end{array}$ & $15(12-21)$ & $15(12-21)$ & $14(12-20)$ & 0.479 \\
\hline $\begin{array}{l}\text { CES-D, median } \\
\text { (interquartile range) }\end{array}$ & $3(1-9)$ & $4(1-10)$ & $3(0-8)$ & 0.885 \\
\hline
\end{tabular}


mean (SD) systolic BP (135.3 (18.7) mmHg; $p<0.01)$ and diastolic BP (79.5 (11.1) $\mathrm{mmHg}$; $p<0.01)$.

\section{Prevalence of diabetes mellitus, depression and psychological distress}

Thirty participants (7.7\%) fulfilled criteria for DM. Of these, 15 (11.9\%) were male and 15 (5.7\%) female. Based on the K10, 109 (28.1\%) participants were found to have psychological distress, with a similar prevalence in men $(n=35,27.8 \%)$ and women $(n=74,28.2 \%)$. A CES-D score of $\geq 16$, indicating mild to moderate depressive symptoms, was found in $60(15.5 \%)$ participants (19 (15.1\%) men and 41 (15.6\%) women).

\section{Association between diabetes, depression and psychological distress}

Table 2 reports the results of the univariate analyses of factors associated with depression and psychological distress. The presence of depression as measured by the CES-D was significantly associated with DM, with an OR of 3.65 (95\% CI $1.64-8.14 ; p=0.002$ ). The presence of psychological distress as measured by the K10 was associated with an almost threefold increase in the odds of diabetes (OR 2.81; 95\% CI 1.32 - 5.96; $p=0.007$ ).

In stepwise logistic regression analysis (Table 3), diabetes remained significantly associated with depression (adjusted OR (AOR) 3.90; 95\% CI 1.33 - 11.37; $p=0.013$ ) and psychological distress (AOR 3.62; $95 \%$ CI $1.31-10.00 ; p=0.013$ ) after controlling for age, gender, BMI, BP, cholesterol and smoking history. Gender, BMI, age, systolic and diastolic BP, serum cholesterol and tobacco smoking did not have a significant effect on the prevalence of psychological distress and depression.

\section{Discussion}

There were several key findings. The prevalence of self-reported psychological distress (28.1\%) and depression (15.5\%) was found to be high. Our prevalence rates were found to be higher than the results obtained in the SASH study, where the prevalence of an MDE was found to be $4.9 \%$ over a 12-month period, the lifetime prevalence being $9.8 \% .{ }^{[3]}$ This may be explained by the high levels of job stress, low salaries and poor support endured by many teachers in the Western Cape. A study by Emsley et al. ${ }^{[13]}$ found that $81 \%$ of their cohort of Cape Town-based teachers identified 'workrelated stress' as a significant causal factor for the development of their psychiatric

Table 2. Association between depression, psychological distress and diabetes mellitus: Univariate analysis

\begin{tabular}{|c|c|c|c|c|}
\hline & \multicolumn{2}{|l|}{ CES-D } & \multicolumn{2}{|l|}{ K10 } \\
\hline & OR (95\% CI) & $p$-value & OR $(95 \% \mathrm{CI})$ & $p$-value \\
\hline Age (years) & $0.96(0.93-0.99)$ & $0.013^{*}$ & $0.96(0.94-0.99)$ & $0.005^{*}$ \\
\hline \multicolumn{5}{|l|}{ Diabetes mellitus } \\
\hline No & Reference & & Reference & \\
\hline Yes & $3.65(1.64-8.14)$ & $0.002^{*}$ & $2.81(1.32-5.96)$ & $0.007^{*}$ \\
\hline \multicolumn{5}{|l|}{ Gender } \\
\hline Male & Reference & & Reference & \\
\hline Female & $1.04(0.58-1.89)$ & 0.884 & $1.02(0.64-1.64)$ & 0.924 \\
\hline BMI $\left(\mathrm{kg} / \mathrm{m}^{2}\right)$ & $1.03(0.99-1.07)$ & 0.196 & $1.01(0.97-1.05)$ & 0.572 \\
\hline Serum cholesterol $(\mathrm{mmol} / \mathrm{L})$ & $0.91(0.69-1.21)$ & 0.514 & $1.06(0.85-1.32)$ & 0.599 \\
\hline Systolic BP (mmHg) & $0.99(0.98-1.01)$ & 0.627 & $0.99(0.97-1.00)$ & 0.050 \\
\hline Diastolic BP (mmHg) & $1.02(0.99-1.05)$ & 0.150 & $0.99(0.97-1.02)$ & 0.690 \\
\hline \multicolumn{5}{|l|}{ Smoking } \\
\hline No & Reference & & Reference & \\
\hline Yes & $1.39(0.71-2.70)$ & 0.333 & $1.42(0.82-2.45)$ & 0.207 \\
\hline
\end{tabular}

Table 3. Association between depression, psychological distress and diabetes mellitus: Multivariate logistic regression analysis

\begin{tabular}{|c|c|c|c|c|}
\hline & \multicolumn{2}{|l|}{ CES-D } & \multicolumn{2}{|l|}{ K10 } \\
\hline & AOR (95\% CI) & $p$-value & AOR (95\% CI) & $p$-value \\
\hline Age (years) & $0.96(0.92-1.01)$ & 0.137 & $0.97(0.93-1.00)$ & 0.080 \\
\hline \multicolumn{5}{|l|}{ Diabetes mellitus } \\
\hline No & Reference & & Reference & \\
\hline Yes & $3.90(1.33-11.37)$ & $0.013^{*}$ & $3.62(1.31-10.00)$ & $0.013^{*}$ \\
\hline \multicolumn{5}{|l|}{ Gender } \\
\hline Male & Reference & & Reference & \\
\hline Female & $1.11(0.50-2.51)$ & 0.405 & $0.98(0.52-1.88)$ & 0.987 \\
\hline BMI $\left(\mathrm{kg} / \mathrm{m}^{2}\right)$ & $1.02(0.97-1.07)$ & 0.532 & $1.01(0.97-1.05)$ & 0.606 \\
\hline $\begin{array}{l}\text { Serum cholesterol } \\
(\mathrm{mmol} / \mathrm{L})\end{array}$ & $0.97(0.66-1.42)$ & 0.780 & $1.06(0.78-1.46)$ & 0.696 \\
\hline Systolic BP (mmHg) & $0.99(0.96-1.01)$ & 0.328 & $0.98(0.96-1.00)$ & 0.106 \\
\hline Diastolic BP (mmHg) & $1.03(0.98-1.07)$ & 0.265 & $1.01(0.97-1.05)$ & 0.585 \\
\hline \multicolumn{5}{|l|}{ Smoking } \\
\hline No & Reference & & Reference & \\
\hline Yes & $0.96(0.41-2.29)$ & 0.944 & $1.15(0.58-2.31)$ & 0.682 \\
\hline
\end{tabular}

disorder. It should also be noted that our cohort differs from the general population in that it consists of a mostly female sample, of relatively young working age and mixed ancestry.

The SASH study found that females had a 1.75 times greater risk than males of experiencing depression, ${ }^{[3]}$ but in this cohort there was no significant difference in the prevalence of either psychological distress or depression in males and females. This may be due to both genders in our cohort being similar in age, and of similar cardiovascular risk, and experiencing the same type of job stress.

The Global Prevalence of Diabetes study estimates the worldwide prevalence of diabetes to be $2.8 \%$ in 2000 and $4.4 \%$ in $2030 .^{[1]}$ A study conducted during 2008 - 2009 found a crude prevalence rate of type $2 \mathrm{DM}$ of 
$28.2 \%$ in a cohort of 642 participants in the Western Cape. ${ }^{[2]}$ This high prevalence rate may be explained by the sample being a general community-based sample and this study having included the use of an oral glucose tolerance test to diagnose type $2 \mathrm{DM}$. The prevalence of DM in our sample was $7.7 \%$. The difference in prevalence rates may further be explained by a possible non-response bias, where known diabetics may have chosen not to participate, or because this sample represented a professional group. After adjusting for confounding variables, DM was associated with a threefold increase in the odds of psychological distress and depression. This is in keeping with other studies that have shown a strong relationship between these two disorders.

\section{Study strengths and limitations}

Strengths of this study include the administration of clinical assessments and validated rating scales in a relatively large cohort of teachers from schools in the Cape Town metropole. Limitations include the lack of generalisability of these findings to other professional and general population groups. Ninety participants were excluded owing to missing mental health data, which may reflect hesitancy to report these symptoms; this may have led to an underreporting of the true prevalence of depression and psychological distress.

\section{Conclusion}

SA teachers were found to have a high prevalence of obesity and DM and a high mean systolic BP. These comorbidities often cluster, increasing the risk of the metabolic syndrome.

Acknowledgments. We acknowledge the PaCT Steering Committee of the Faculty of Medicine and Health Sciences at Stellenbosch University for their guidance with the SA pilot study and the primary collaborators of the Africa/Harvard School of Public Health Partnership for Cohort Research and Training for their efforts in initiating, carrying out, and co-ordinating this study in five sites (other than SA): Clement Adebamowo (Nigeria site), Marina Njelekela (Tanzania site), David Guwatudde (Makerere, Uganda site), Francis Bajunirwe (Mbarara, Uganda site), Hans-Olov Adami, Shona Dalal, Michelle Holmes and Todd Reid (HSPH co-ordinating center). The SA pilot study was made possible through funding obtained from the Dean's Office of HSPH, and the HSPH Department of Nutrition; the Faculty of Medicine and Health Sciences at Stellenbosch University; and the International Society of Nephrology's Global Outreach Clinical Research and Prevention Program.

\section{References}

1. Wild S, Roglic G, Green A, et al. Global prevalence of diabetes: Estimates for the year 2000 and projections for 2030. Diabetes Care 2004;27(5):1047-1053. [http://dx.doi.org/10.2337/ diacare.27.5.1047

2. Erasmus RT, Soita DJ, Hassan MS, et al. High prevalence of diabetes mellitus and metabolic syndrome in a South African coloured population: Baseline data of a study in Bellville, Cape Town. S Afr Med I 2012;102(11):841-844. [http://dx.doi.org/10.7196/SAMJ.5670]

3. Tomlinson M, Grimsrud AT, Stein DJ, et al. The epidemiology of major depression in South Africa: Results from the South African Stress and Health study. S Afr Med J 2009;99(5):368-373.

4. Mezuk B, Eaton WW, Albrecht S, et al. Depression and type 2 diabetes over the lifespan: A metaanalysis. Diabetes Care 2008;31(12):2383-2390. [http://dx.doi.org/10.2337/dc08-0985]

5. Anderson RJ, Freedland KE, Clouse RE, et al. The prevalence of comorbid depression in adults with diabetes A meta-analysis. Diabetes Care 2001;24(6):1069-1078. [http://dx.doi.org/10.2337/diacare.24.6.1069]

6. Knol M, Twisk J, Beekman A, Heine R, Snoek F, Pouwer F. Depression as a risk factor for the onse of type 2 diabetes mellitus. A meta-analysis. Diabetologia 2006;49(5):837-845. [http://dx.doi. org/10.1007/s00125-006-0159-x]

7. Mayosi BM, Flisher AJ, Lalloo UG, Sitas F, Tollman SM, Bradshaw D. The burden of noncommunicable diseases in South Africa. Lancet 2009;374(9693):934-947. [http://dx.doi.org/10.1016/ S0140-6736(09)61087-4]

8. Peltzer $\mathrm{K}$, Shisana $\mathrm{O}$, Zuma $\mathrm{K}$, van Wyk B, Zungu-Dirwayi N. Job stress, job satisfaction and stressrelated illnesses among South African educators. Stress Health 2009;25(3):247-257.

9. World Health Organization. STEPwise approach to noncommunicable disease risk factor surveillance World Health Organization. STEPwise approach to noncommunicable disease risk factor surveillance
(STEPS). Geneva: World Health Organization, 2015. http://www.who.int/chp/steps/riskfactor/en/ (accessed 20 October 2015).

10. Radloff LS. The CES-D scale: A self-report depression scale for research in the general population. Appl Psychol Meas 1977;1(3):385-401. [http://dx.doi.org/10.1177/014662167700100306]

1. Andrews G, Slade T. Interpreting scores on the Kessler psychological distress scale (K10). Aust N Z Public Health 2001;25(6):494-497. [http://dx.doi.org/10.1111/j.1467-842X.2001.tb00310.x]

12. Jorm AF. Does old age reduce the risk of anxiety and depression? A review of epidemiological studies across the adult life span. Psychol Med 2000;30(1):11-22. [http://dx.doi.org/10.1017/ S0033291799001452]

13. Emsley R, Emsley L, Seedat S. Occupational disability on psychiatric grounds in South African schoolteachers. Afr J Psychiatry 2009;12(3):223-226. [http://dx.doi.org/10.4314/ajpsy.v12i3.48498]

Accepted 5 October 2015 